\title{
Perturbation study of nonequilibrium quasi-particle spectra in an infinite-dimensional Hubbard lattice
}

\author{
R. J. Heary and J. E. Han \\ Department of Physics, State University of New York at Buffalo, Buffalo, NY 14260, USA
}

(Dated: June 17, 2022)

\begin{abstract}
A model for nonequilibrium dynamical mean-field theory is constructed for the infinite dimensional Hubbard lattice. We impose nonequilibrium by expressing the physical orbital as a superposition of a left- $(L)$ moving and right- $(R)$ moving electronic state with the respective chemical potential $\mu_{L}$ and $\mu_{R}$. Using the second-order iterative perturbation theory we calculate the quasi-particle properties as a function of the chemical potential bias between the $L$ and $R$ movers, i.e. $\Phi=\mu_{L}-\mu_{R}$. The evolution of the nonequilibrium quasi-particle spectrum is mapped out as a function of the bias and temperature. The quasi-particle states with the renormalized Fermi energy scale $\varepsilon_{Q P}^{0}$ disappear at $\Phi \sim \varepsilon_{Q P}^{0}$ in the low temperature limit. The second-order perturbation theory predicts that in the vicinity of the Mott-insulator transition at the Coulomb parameter $U=U_{c}$, there exist another critical Coulomb parameter $U_{d}\left(<U_{c}\right)$ such that, for $U_{d}<U<U c$, quasi-particle states are destroyed discontinuously when $\left(\varepsilon_{Q P}^{0}\right)^{2} \sim a\left(\pi k_{B} T_{c}\right)^{2}+b \Phi_{c}^{2}$ with the critical temperature $T_{c}$ and the critical bias $\Phi_{c}$.

PACS numbers: 71.10.-w, 71.10.Fd, 71.30.+h, 73.40.Jn
\end{abstract}

In recent years significant experimental progress has been made in the fabrication of sophisticated electronic heterostructures utilizing strongly correlated electronic materials. These systems have given rise to the discovery of rich novel phenomena. These include: ballistic transport of electrons through heterostructures of superconductors $^{11}$, ferromagnets ${ }^{2}$, magnetic tunneling junctions $s^{3 / 4}$ and oxides 516178 . With this vast array of strongly correlated heterostructures it is very important to theoretically understand how the strongly correlated materials in the bulk limit will behave under nonequilibrium conditions. In this work we formulate the nonequilibrium problem in the lattice and present significant progress towards a more complete understanding of strongly correlated lattices out of equilibrium.

We are interested in heterostructures of strongly correlated materials under a finite source-drain bias where the voltage drop occurs mainly at the interface of the strongly correlated material and the source/drain leads 910 . Inside the strongly correlated material the transport is ballistic and driven by the momentum distribution of imbalanced chemical potentials. Our focus here is to investigate the evolution of strongly correlated quasi-particle spectra due to nonequilibrium driven by an imbalance in the chemical potentials. In this work we gain qualitative understanding on how the enhanced dephasing by the new particlehole decay channel due to nonequilibrium modifies the spectral properties.

One of the most practical and powerful theoretical techniques for studying strongly correlated lattices is the dynamical mean-field theory (DMFT) $)^{11}$. Within this approach the self-energy of the strongly correlated lattice becomes momentum-independent and as a result the problem is reduced to solving a self-consistent interacting impurity model. This inherent simplicity of DMFT makes it a very attractive tool, especially with the large quantity of exact and perturbative impurity solvers such as the Hirsch-Fye quantum Monte Carlo method ${ }^{12}$, the numerical renormalization group ${ }^{13}$, and the non-crossing approximation $\frac{14] 15}{}$.

As an extension of DMFT to nonequilibrium situations, Okamoto has theoretically studied the nonlinear transport and spectral properties of metal-Mott insulator-metal heterostructures where a bias voltage is applied across the Mott insulator $\frac{910]}{10}$ using layeredDMFT 16. There he combined the layered-DMFT technique with the Keldysh Green function approach and invoked the non-crossing approximation as the impurity solver. Another system recently studied is the DMFT limit of the Hubbard model with the nonequilibrium driven by a uniform high electric field 17 . Others have looked at the DMFT limit of the Falicov-Kimball model in the presence of a uniform time-dependent electric field and examined the transient current, quenching of the Bloch oscillations, and evolution of the spectral function $18 \mid 19$.

In our analysis of the problem we choose to start from the metallic state and analyze how the Fermi liquid is renormalized and eventually destroyed with multiple chemical potentials. We find that the quasi-particles strongly depend upon the strength of the chemical potential difference $\Phi$. At zero $\Phi$ it has long been known that the system undergoes a metal-insulator transition at $U=U_{c}^{11}$. The second order iterative perturbation theory (IPT) approximation gives $U_{c}=U_{c 2} \simeq 3.3 D$ at zero temperature, where $D$ is the half bandwidth. As the temperature is raised the transition remains distinct down to $U_{c 1} \simeq 2.6 D$. In nonequilibrium, the second order IPT shows that the quasi-particles are destroyed abruptly by the chemical potential bias in the region $U_{d}<U<U_{c}$, where $U_{d} \simeq 2.3 D$. When $U<U_{d}$ the system exhibits a smooth crossover from a system of well-defined quasiparticle states to that without quasi-particle excitations.

We start with a non-interacting $d$-dimensional tight- 
binding Bethe lattice. The tight-binding solutions are grouped evenly into left- $(L)$ and right- $(R)$ movers with chemical potentials $\mu_{L}=\Phi / 2$ and $\mu_{R}=-\Phi / 2$, respectively. Since we do not study transport properties in this work, it is not important how the physical site is decomposed into $L, R$ movers. The original site orbital $d_{i \sigma}^{\dagger}$ at site $i$ with spin $\sigma$ is written as a superposition of $L$ and $R$ orbitals $d_{L / R, i \sigma}^{\dagger}$ according to

$$
d_{i \sigma}^{\dagger}=\frac{1}{\sqrt{2}}\left(d_{L, i \sigma}^{\dagger}+d_{R, i \sigma}^{\dagger}\right)
$$

The interaction terms and any observable quantities ought to be given in the physical basis $d_{i \sigma}^{\dagger}$. The nonequilibrium DMFT lattice is depicted in Figure 1 .

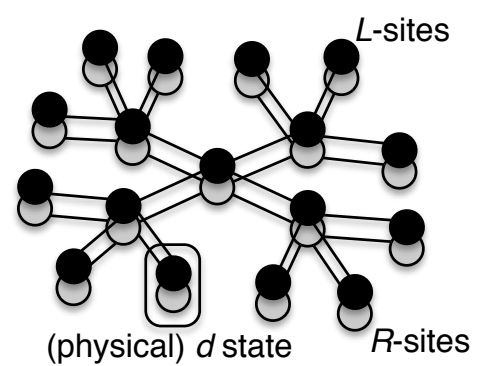

FIG. 1: The Bethe lattice where the (physical) $d$-state is given as a superposition of a left- $(L)$ and a right- $(R)$ moving state with the corresponding chemical potentials $\mu_{L}=\Phi / 2$ and $\mu_{R}=-\Phi / 2$.

The model with which we focus is the DMFT limit $(d \rightarrow \infty)$ of the Hubbard model $\hat{\mathcal{H}}=\hat{\mathcal{H}}_{0}+\hat{\mathcal{V}}$,

$$
\begin{aligned}
\hat{\mathcal{H}}_{0} & =-t \sum_{<i, j>} \sum_{\alpha=L, R ; \sigma}\left(d_{\alpha, i \sigma}^{\dagger} d_{\alpha, j \sigma}+\text { h.c. }\right) \\
\hat{\mathcal{V}} & =U \sum_{i}\left(d_{i \uparrow}^{\dagger} d_{i \uparrow}-\frac{1}{2}\right)\left(d_{i \downarrow}^{\dagger} d_{i \downarrow}-\frac{1}{2}\right) .
\end{aligned}
$$

The non-interacting tight-binding part of the Hamiltonian $\hat{\mathcal{H}}_{0}$ takes into account the hopping of electrons between nearest neighbor lattice sites of the Bethe lattice. The interaction term of the Hamiltonian $\hat{\mathcal{V}}$ is the particle-hole symmetric on-site Coulomb interaction of strength $U$. Both the non-interacting and interacting terms of the Hamiltonian are homogeneous in real space, which is necessary for our application of singlesite DMFT. The Hamiltonian may be further simplified by transforming the $L$ and $R$ basis into the even $(E)$ and odd $(O)$ superposition of the basis. The creation operators for the even and odd electron orbitals are $d_{E / O, i \sigma}^{\dagger}=\left(d_{L, i \sigma}^{\dagger} \pm d_{R, i \sigma}^{\dagger}\right) / \sqrt{2}$. The interaction is only given by the even (physical) basis and the noninteracting part is decoupled into the even-odd parts as $\hat{\mathcal{H}}_{0}=-t \sum_{<i, j>} \sum_{\alpha=E, I ; \sigma}\left(d_{\alpha, i \sigma}^{\dagger} d_{\alpha, j \sigma}+\right.$ h.c. $)$. Therefore, the time-evolution of even (physical) and odd states are completely decoupled and the perturbation theory of DMFT is applied within the physical basis where the nonequilibrium statistics are imposed on the noninteracting Green functions.

This Hamiltonian is solved using the iterative perturbation theory 11 . The IPT has been extensively utilized and well-established in equilibrium DMFT and we anticipate that the second order IPT gives a qualitatively reasonable description of the quasi-particle destruction in nonequilibrium. However we caution that the second order perturbation theory may not capture the exact nature of the quasi-particles in nonequilibrium. For example, it is known that second-order perturbation theory does not properly describe the splitting of the Kondo peak in quantum dot devices with a finite voltage bias, as seen in the fourth-order perturbation theory ${ }^{20}$ and in nonperturbative solvers ${ }^{21 / 22}$. Therefore, it still remains to be seen through nonperturbative calculations which of the following results will hold up.

Now we outline the DMFT self-consistent routine. The calculation is performed in real-time as opposed to realfrequency because close to the transition of a system with well defined quasi-particle states to a system with none the bath spectral function becomes sharply peaked at the Fermi energy ${ }^{23}$, thus making the real-frequency calculation much more difficult. A typical time bandwidth we use is $\sim 3000$. In the non-interacting limit the (even) bath green function is given by

$$
\left[\begin{array}{l}
g^{<}(t) \\
g^{>}(t)
\end{array}\right]=\frac{i}{2} \sum_{\alpha=L, R} \int d \epsilon D(\epsilon)\left[\begin{array}{c}
f_{\alpha}(\epsilon) \\
f_{\alpha}(\epsilon)-1
\end{array}\right] e^{-i \epsilon t}
$$

where $f_{L}(\epsilon)=f\left(\epsilon-\frac{\Phi}{2}\right)$ and $f_{R}(\epsilon)=f\left(\epsilon+\frac{\Phi}{2}\right)$ are the Fermi functions for the $L$ and $R$ movers. $D(\epsilon)$ is the non-interacting density of states (DOS), which we have taken to be semi-circular $D(\epsilon)=\frac{2}{\pi D^{2}} \sqrt{D^{2}-\epsilon^{2}}$. In the following, $D=1$ is used as the unit of energy.

Within IPT the self energy is calculated to secondorder in $U$. Using the Langreth theorem ${ }^{24}$ we may calculate the lesser and greater self energies according to

$$
\Sigma_{\text {int }}^{\gtrless}(t)=U^{2}\left[g^{\gtrless}(t)\right]^{2} g^{\lessgtr}(-t) .
$$

The lesser and greater self energies are then Fourier transformed to real frequency. The lesser (greater) self energy gives us the particle (hole) spectral weight as a function of frequency for the retarded self-energy. Using the Keldysh Green function relation $\Sigma_{\text {int }}^{r}(\omega)-\Sigma_{\text {int }}^{a}(\omega)=$ $\Sigma_{\text {int }}^{>}(\omega)-\Sigma_{\text {int }}^{<}(\omega)$, we may express the retarded self energy in the spectral form

$$
\Sigma_{\text {int }}^{r}(\omega)=\frac{1}{2 \pi} \int d \epsilon \frac{\operatorname{Im}\left[\Sigma_{\text {int }}^{<}(\epsilon)-\Sigma_{\text {int }}^{>}(\epsilon)\right]}{\omega-\epsilon+i \eta} .
$$

The nonequilibrium DMFT self consistent equations for the interacting and bath Green function are therefore

$$
\begin{aligned}
G^{r}(\omega) & =\int d \epsilon \frac{D(\epsilon)}{\omega-\epsilon-\Sigma_{i n t}^{r}(\omega)} \\
g^{r}(\omega)^{-1} & =\omega+i \eta-t^{2} G^{r}(\omega) .
\end{aligned}
$$


where $\rho_{0}(\omega)=-\pi^{-1} \operatorname{Im}\left[g^{r}(\omega)\right]$ is the new bath DOS. For the new Keldysh bath Green functions we replace the non-interacting DOS in Eq. (4) with $\rho_{0}(\omega)$. The momentum independence of the self energy and the translational invariance ensure that the wave vectors remain good quantum numbers. We iterate these equations until the bath Green function and interacting Green function converge.

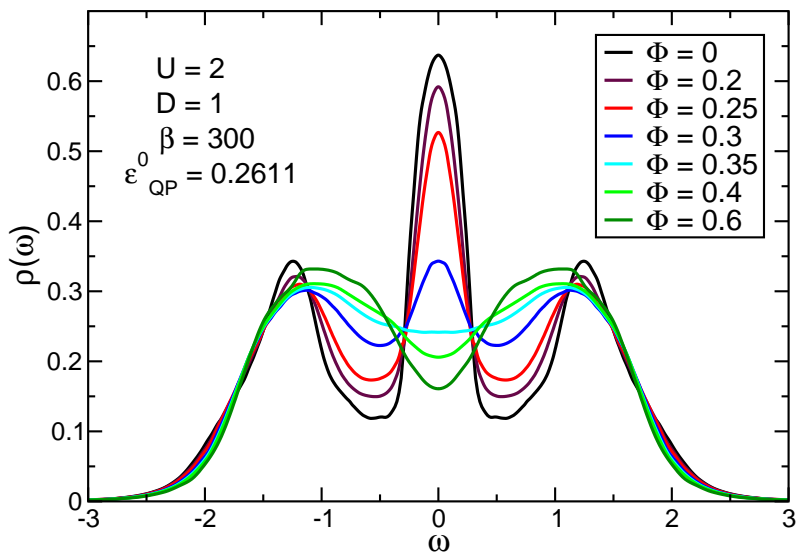

FIG. 2: (color online) Interacting local spectral functions for $U=2$, inverse temperature $\beta=300$ and half bandwidth $D=1$ plotted for a range of chemical potential biases, $\Phi$. At this value of the Coulomb interaction $U$ the quasi-particle peak is destroyed continuously as $\Phi$ is increased.

From previous works in the equilibrium system $\frac{11}{11}$, it is known that by increasing the temperature the quasiparticles are destroyed when the thermal fluctuations surpass a certain low-energy scale. This low-energy scale is given by the renormalized Fermi energy $\varepsilon_{Q P}$ which we define as the half-width at half maximum (HWHM) of the quasi-particle peak and is similar to $\epsilon_{F}^{*}=Z D$ where $Z=\left[1-\left.\partial_{\omega} \operatorname{Re}\left[\Sigma^{r}(\omega)\right]\right|_{\omega=0}\right]^{-1}$ is the quasi-particle weight, as found in Georges et.a!11. As the temperature is increased at zero bias $\Phi=0$, the quasi-particles are destroyed when $k_{B} T_{c} \sim \varepsilon_{Q P}^{0}$ where $T_{c}$ is the critical temperature. $\varepsilon_{Q P}^{0}$ is the quasi-particle half bandwidth at zero bias.

Figures 2 4 show the evolution of quasi-particle spectra as a function of bias $\Phi$. The destruction of the Fermi liquid is easily understood by enhanced dephasing of particles and holes, which is a consequence of the opening up of phase space available for the particles/holes to scatter into through $\Phi$ and by thermal fluctuations. Both of these effects lead to a finite lifetime for the electron at the Fermi energy. To lowest order in $\omega, T$ and $\Phi$ the imaginary part of the self energy obeys the relation 25

$$
\lim _{\omega, T, \Phi \rightarrow 0} \operatorname{Im}\left[\Sigma^{r}(\omega)\right] \propto\left[\omega^{2}+\left(\pi k_{B} T\right)^{2}+\frac{3}{4} \Phi^{2}\right] .
$$

The interacting spectral function $\rho(\omega)$ is calculated both as a function of the Coulomb interaction $U$ and the applied chemical potential bias $\Phi$. For $U<U_{d}$ with

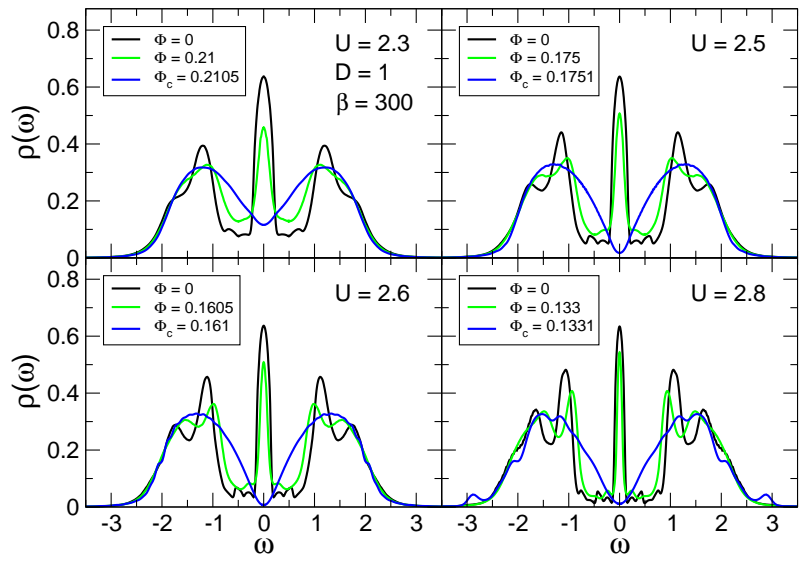

FIG. 3: (color online) Interacting local spectral functions for $U=2.3,2.5,2.6$, and 2.8 at inverse temperature $\beta=300$ and band width $D=1$. These plots depict the sudden disappearance of the quasi-particle peak at the critical voltage $\Phi_{c}$. The spectral functions are plotted at $\Phi=0$, at $\Phi$ just before the quasi-particle destruction, and at $\Phi=\Phi_{c}$.

$U_{d} \simeq 2.3 D$, the destruction of the quasi-particle peak is continuous, and exhibits similar behavior to the metalinsulator transition in the crossover regime $\left(U<U_{c 1}\right)$ of equilibrium DMFT. Our results at $U=2 D\left(<U_{d}\right)$ are shown in Figure 2. As the chemical potential bias is increased the quasi-particle weight smoothly shifts towards the upper and lower Hubbard bands, and the quasiparticle peak disappears when $\Phi \sim \varepsilon_{Q P}^{0}$.

For $U_{d}<U<U_{c}$ the quasi-particle peak is discontinuously destroyed at a critical chemical potential bias $\Phi_{c}$. In Figure 3 we have plotted the interacting spectral functions for $U / D=2.3,2.5,2.6$, and 2.8 to illustrate the sudden disappearance of the quasi-particle peak. The spectral function for each value of $U$ is shown for $\Phi=0$, $\Phi$ just before the transition, and finally at the transition $\Phi=\Phi_{c}$. This discontinuous transition in nonequilibrium by bias is reminiscent of the discontinuous transition by temperature in the equilibrium DMFT.

In Figure 4 the scaled quasi-particle energy $\varepsilon_{Q P} / \varepsilon_{Q P}^{0}$ is plotted versus the scaled chemical potential bias $\Phi / \Phi_{c}$. For $\Phi \lesssim 0.4 \Phi_{c}$ the curves for $U / D=2.3,2.5,2.6$ and 2.8 scale onto a single curve. At $\Phi=\Phi_{c}$ it is clearly visible that the quasi-particle peak disappears discontinuously for each value of $U$.

In Figure 5 we take a closer look at the temperature dependence of the transition in the discontinuous region. At $U=2.8$, which is well into this region, the quasiparticle disappearance remains discontinuous for the entire range of temperatures. Therefore the discontinuous nature of the transition is very robust and is not effected by the temperature. The inset yields the dependence of the critical bias upon temperature. Motivated by the form of the imaginary part of the self energy [Eq. (9)], we anticipate that the destruction of the quasi-particles will occur when $\left(\varepsilon_{Q P}^{0}\right)^{2} \sim a\left(\pi k_{B} T_{c}\right)^{2}+b \Phi_{c}^{2}$ at critical values for the temperature $T_{c}$ and the bias $\Phi_{c}$. In Figure 5 we 


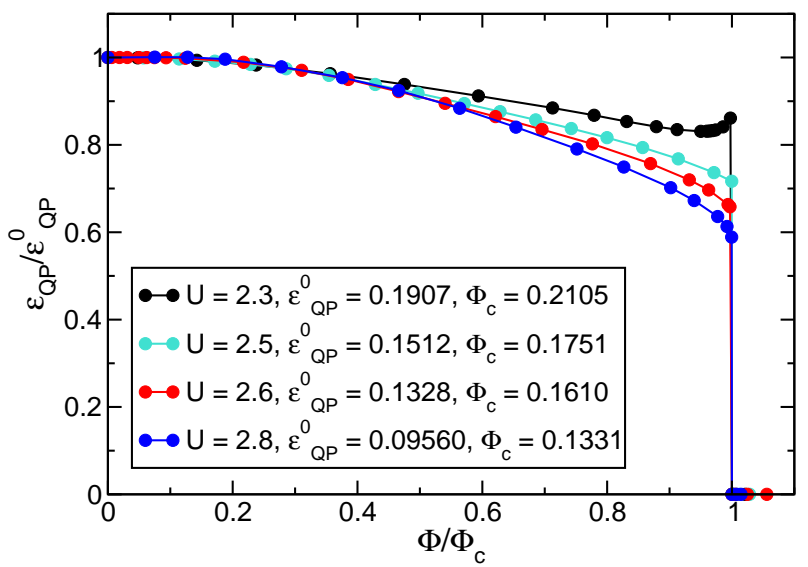

FIG. 4: (color online) Quasi-particle energy $\varepsilon_{Q P}$ as a function of the chemical potential bias $\Phi$ at $\beta=300$ and $D=1$. The quasi-particle energy is scaled to the quasi-particle energy at zero bias, $\varepsilon_{Q P}^{0}$, and the chemical potential bias is scaled to the critical bias at which the quasi-particle peak is destroyed, $\Phi_{c}$. For small bias, $\Phi \lesssim 0.4 \Phi_{c}$, the quasi-particle energies scale to a single curve. In this range of values for $U$ the disappearance of the quasi-particle peak is strongly discontinuous.

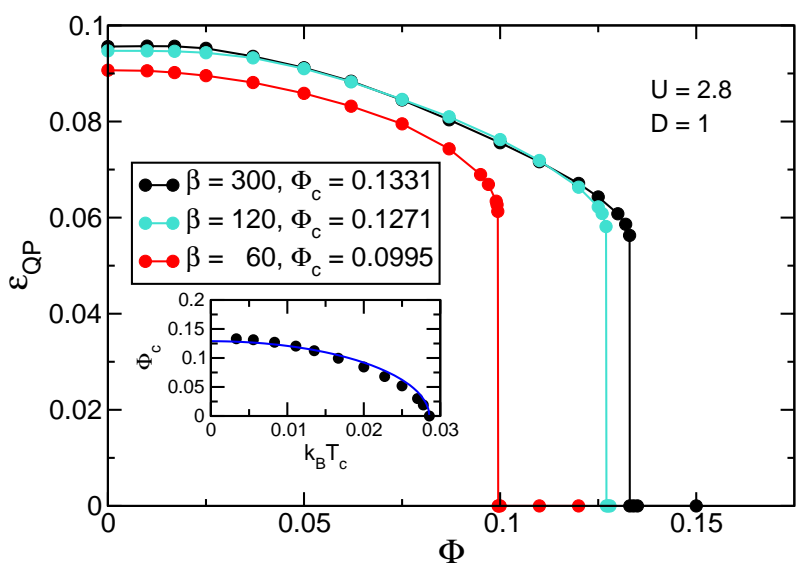

FIG. 5: (color online) Quasi-particle energy $\varepsilon_{Q P}$ as a function of the chemical potential bias $\Phi$ at $U=2.8$ and $D=1$ for different values of the inverse temperature $\beta$. As the temperature is increased, the disappearance of quasi-particles remains discontinuous and the critical bias $\Phi_{c}$ is lowered. The inset gives the temperature dependence of the critical bias. The data (black circles) was fit (blue curve) to the function, $\left(\varepsilon_{Q P}^{0}\right)^{2} \sim a\left(\pi k_{B} T_{c}\right)^{2}+b \Phi_{c}^{2}$, where $a=1.1$ and $b=0.54$.

fit $\Phi_{c}$ versus $T_{c}$ to this function and find that $a=1.1$ and $b=0.54$. Considering the nonlinear effects in the numerical results, the obtained values $a$ and $b$ are in reasonable agreement with the estimates 1 and $\frac{3}{4}$ based on Eq. (9).
The critical bias $\Phi_{c}$ and renormalized Fermi energy $\varepsilon_{Q P}^{0}$ are plotted versus $U$ in Figure 6 at $\beta=300$. The onset of the discontinuous transition is marked at $U_{d} \simeq$ $2.3 D$ and the Mott-insulator transition at $\Phi=0$ is given at $U_{c} \simeq 3.3 D$. We see that the transition occurs at $\Phi_{c} \sim$

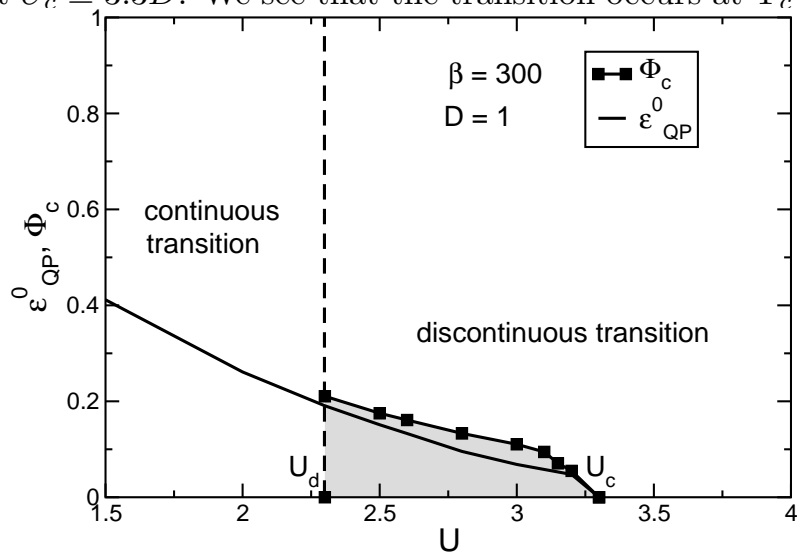

FIG. 6: The critical bias $\Phi_{c}$ is plotted as a function of the Coulomb interaction $U$ at inverse temperature $\beta=300$ and bandwidth $D=1$. We have also plotted the renormalized Fermi energy $\varepsilon_{Q P}^{0}$ at $\Phi=0$. In the region $U_{d}<U<U_{c}$ the quasi-particle peak is destroyed discontinuously at $\Phi_{c}$. When $U<U_{d}$ the quasi-particle peak disappears smoothly with increasing chemical potential bias. At this temperature $\Phi_{c} \sim \varepsilon_{Q P}^{0}$.

$\varepsilon_{Q P}^{0}$ which further justifies the function used for fitting $\Phi_{c}$ versus $k_{B} T_{c}$ of Figure 5 .

In this work we have presented a theoretical model for nonequilibrium dynamical mean-field theory and performed the iterative perturbation calculations within the dynamical mean-field theory. In a lattice where the statistics are determined by the presence of multiple chemical potentials, the quasi-particle properties are strongly dependent upon the strength of the Coulomb interaction and the chemical potential bias, $\Phi$. For $U<U_{d}$ with $U_{d} \simeq 2.3 D$ for the bandwidth $D$, the quasi-particle disappears smoothly with increasing chemical potential bias. The disappearance of the quasi-particles is caused by the opening up of phase space for the electrons to scatter into at the Fermi energy, resulting in a finite lifetime for the electrons. The perturbation theory also predicts that in the region $U_{d}<U<U_{c}$, where $U_{c} \simeq 3.3 D$ marks the Mott-insulator transition in equilibrium, the quasiparticle particles may be destroyed discontinuously at a critical bias, $\Phi_{c}$.

This work was supported by NSF DMR-0426826 and we acknowledge the CCR at the SUNY Buffalo for computational resources.
1 G. Bastian, E. O. Göbel, A. B. Zorin, H. Schulze, J. Niemeyer, and T. Weimann, Phys. Rev. Lett. 81, 1686
(1998).

2 J. Salafranca, M. J. Calderón, and L. Brey, Phys. Rev. B 
77, 014441 (2008).

3 J. M. De Teresa, A. Barthélémy, A. Fert, J. Contour, F. Montaigne, P. Seneor, Science 286, 507 (1999).

4 M. R. McCartney, R. E. Dunin-Borkowski, M. R. Scheinfein, D. J. Smith, S. Gider, S. Parkin, Science 286, 1337 (1999).

5 S. Thiel, G. Hammerl, A. Schmehl, C. W. Schneider, J. Mannhart, Science 313, 1942 (2006).

6 J. Chakhalian, J. W. Freeland, G. Srajer, J. Strempfer, G. Khaliullin, J. C. Cezar, T. Charlton, R. Dalgliesh, C. Bernhard, G. Cristiani, H. -U. Habermeier, and B. Keimer, Nature Phys. 2, 244 (2006).

7 A. Brinkman, M. Huijben, M. van Zalk, J. Huijben, U. Zeitler, J. C. Maan, W. G. van der Wiel, G. Rijnders, D. H. A. Blank, and H. Hilgenkamp, Nature Mater. 6, 493 (2007).

8 N. Reyren, S Thiel, A. D. Caviglia, L. F. Kourkoutis, G. Hammerl, C. Richter, C. W. Schneider, T. Kopp, A. -S. Rüetschi, D. Jaccard, M. Gabay, D. A. Muller, J. -M. Triscone, J. Mannhart, Science 31, 1196 (2007).

9 S. Okamoto, Phys. Rev. B 76, 035105 (2007).

10 S. Okamoto, Phys. Rev. Lett. 101, 116807 (2008).

11 A. Georges, G. Kotliar, W. Krauth, M. J. Rozenberg, Rev. Mod. Phys. 68, 13 (1996).

12 J. E. Hirsch, R. M. Fye, Phys. Rev. Lett. 56, 2521 (1986).
${ }^{13}$ H. R. Krishna-murthy, J. W. Wilkins, and K. G. Wilson, Phys. Rev. B 21, 1003 (1980); ibid. 21, 1044 (1980).

14 T. Pruschke, N. Grewe, Z. Phys. B 74, 439 (1993).

15 T. Pruschke, D. L. Cox, M. Jarrell, Phys. Rev. B 47, 3553 (1989).

16 S. Okamoto and A. J. Millis, Phys. Rev. B 70, 241104 (2004).

17 A. V. Joura, J. K. Freericks, Th. Pruschke, Phys. Rev. Lett. 101, 196401 (2008).

18 J. K. Freericks, V. M. Turkowski, V. Zlatic, Phys. Rev. Lett. 97, 266408 (2006).

19 J. K. Freericks, Phys. Rev. B 77, 075109 (2008).

20 T. Fujii and K. Ueda, Phys. Rev. B 68, 155310 (2003).

21 J. E. Han and R. J. Heary, Phys. Rev. Lett. 99, 236808 (2007).

${ }^{22}$ F. B. Anders, Phys. Rev. Lett. 101, 066804 (2008).

23 X. Y. Zhang, M. J. Rozenberg, and G. Kotliar, Phys. Rev. Lett. 70, 1666 (1993).

24 D. C. Langreth, in Linear and Nonlinear Electron Transport in Solids, Vol. 17 of Nato Advanced Studies Institute, Series B: Physics, edited by J. T. Devreese and V. E. Doren (Plenum, New York, 1976).

25 Akira Oguri, Phys. Rev. B 64, 153305 (2001). 